\title{
Erratum to: Durability of Composites in a Marine Environment 2
}

Peter Davies and Yapa D.S. Rajapakse

\section{Erratum to:}

P. Davies and Y.D.S. Rajapakse (eds.), Durability of Composites in a Marine Environment 2, Solid Mechanics and Its Applications 245, https://doi.org/10.1007/978-3-319-65145-3

In the original version of the book, series volume number has been changed from 244 to 245 . The erratum book has been updated with the change.

The updated online version of this book can be found at https://doi.org/10.1007/978-3-319-65145-3 